\title{
Non-isothermal Curing Kinetics of Modified Amine-Epoxy Asphalt
} Jingbo MAO ${ }^{1, a}$, Dejin $\mathrm{CHENG}^{2, \mathrm{~b}}$, Jie WANG ${ }^{2, \mathrm{c}}$, Jiale SONG ${ }^{2, \mathrm{~d}}$, Weiguang $\mathrm{Li}^{3, \mathrm{e}}$

${ }^{1}$ Eastern Alliance Construction Engineering Co., Ltd, CCCC Second Highway Engineering Co., Ltd., Xi'an, 710119, China

\author{
${ }^{2}$ School of Materials Science and Engineering, Chang' an University, Xi'an, 710064, China \\ ${ }^{3}$ School of Highway, Chang' an University, Xi'an, 710064, China \\ aemail: fluiway@163.com, bemail: chengdj1991@163.com, 'email: 1159280067@qq.com, 'email: \\ jlsong@chd.edu.cn, eemail:wgli@chd.edu.cn
}

Keywords: Epoxy asphalt; Curing reaction; Self-catalytic; Activation energy; Diffusion

\begin{abstract}
The curing reaction characteristics of modified amine-epoxy and epoxy asphalt system under different heating rates were analyzed by differential scanning calorimetry (DSC). The relationship between curing degree and activation energy during the curing reaction is studied. The Kissinger equation was used to calculate the apparent activation energy of the reaction, and the rate constant and the reaction order of the curing reaction were fitted by Kamal equation. The results showed that the curing reaction was a typical self-catalytic reaction, the apparent activation energy of epoxy system and epoxy asphalt system were $34.95 \mathrm{~kJ} / \mathrm{mol}$ and $42.02 \mathrm{~kJ} / \mathrm{mol}$, respectively. Meanwhile the curing reaction rate constant and order of the epoxy asphalt system were less than those of the epoxy system. The curing reaction rate of epoxy curing system was contracted by introduced asphalt. Furthermore, the late of curing reaction was dominated by diffusion control.
\end{abstract}

\section{Introduction}

Asphalt is a very widely used material in road and bridge construction. However, it soften easily at high temperature and brittle at low temperature because of its special chemical composition and molecular structure. Polymer is usually used to improve the performance of asphalt [1], such as SBS, SBR, EVA and natural rubber, but the thermoplastic properties of asphalt are not changed. Epoxy asphalt, which is composed of epoxy resin, curing agent, asphalt and additives, is a kind of revolutionary upgrade material. After curing, epoxy asphalt becomes into an irreversible three-dimensional thermosetting composite. The asphalt is firmly bound up in the cross-linked network of epoxy resin. The thermal plastic behavior of that is changed fundamentally and the road performance of the asphalt is improved significantly [2-3].

When the epoxy resin and the curing agent are mixed at a certain temperature, curing reaction begins to occur. The viscosity of epoxy asphalt system increases with time gradually, but after the reaction to a certain extent, the viscosity of the system increases suddenly to form a gel, and loss of fluidity. The curing reaction of epoxy asphalt is very complex. However, in actual construction process, especially selection of mixing temperature and the determination of paving and rolling time, are closely related to the curing reaction of epoxy asphalt. Therefore, it is necessary to study the curing reaction mechanism and rules of epoxy asphalt, so as to provide a theoretical basis for the optimization of the construction technology parameters [4].

Study on reaction kinetics of solid materials by thermal analysis technique in the condition of constant or variable temperature. Thus forming a kind of "non isothermal kinetics" branch, which is widely used in various fields [5-6]. In this paper, the curing onset temperature, peak temperature, and offset temperature were analyzed by DSC during curing process of the modified amine epoxy asphalt and epoxy system. The Kissinger equation was used to calculate the apparent activation energy of the curing system, and the rate constant and reaction order of the curing reaction were fitted by Kamal equation of the self-catalytic reaction model. The curing reaction is thoroughly studied, which provides theoretical guidance for the further research of curing mechanism. 


\section{Experimental}

\subsection{Raw Materials}

The main properties of Shell 90\# road petroleum asphalt meet the requirements of the relevant technical requirements of the road petroleum asphalt in China; Epoxy resin from Wuxi resin factory is E-51 Bisphenol A epoxy resin (Epoxy equivalent is $190 \mathrm{~g} / \mathrm{mol}, 12500 \mathrm{mPa} \cdot \mathrm{s}$ at $25^{\circ} \mathrm{C}, 2400$ $\mathrm{mPa} \cdot \mathrm{s}$ at $40^{\circ} \mathrm{C}$ ); Modified amine curing agent was made by laboratory (Amine equivalent is 100 $\mathrm{g} / \mathrm{mol}, 110 \mathrm{mPa} \cdot \mathrm{s}$ at $25^{\circ} \mathrm{C}$ ) which has low reactivity and good compatibility with asphalt and epoxy resin; In order to meet the application requirements, epoxy toughening agent of sale was joined in curing formula.

\subsection{Method}

The epoxy resin modified with active toughening agent was mixed with the modified amine curing agent by mass ratio 2:1, and the epoxy group with active hydrogen molar ratio of approximately 1:1. The sample about $10 \mathrm{mg}$ was put in a DSC dish after mixing evenly. The curing process of the sample was determined by differential scanning calorimetry of DSC-7 from PE Company. The high purity nitrogen was used as test atmosphere, and the heating rates were $5{ }^{\circ} \mathrm{C} / \mathrm{min}, 10{ }^{\circ} \mathrm{C} / \mathrm{min}, 15{ }^{\circ} \mathrm{C} / \mathrm{min}$ and $20{ }^{\circ} \mathrm{C} / \mathrm{min}$ respectively. Test temperature range from $25{ }^{\circ} \mathrm{C} \sim 300{ }^{\circ} \mathrm{C}$. In the above epoxy curing formula, the epoxy resin and asphalt were mixed by the mass ratio of $1: 1$. After mixing evenly, the sample was about $10 \mathrm{mg}$, and the same method was used to carry out the DSC heating test.

\section{Results and Discussion}

\subsection{Non- isothermal DSC Scan Results}

The four heating rate DSC curves of epoxy and epoxy asphalt were shown in Fig. 1. From Fig. 1 we could saw that the exothermic peak of the curve gradually moves to the high temperature zone with the increase of heating rate, and the curing time was shortened. In order to achieve the best state for the curing reaction, the maximum temperature of exothermic peak, required a certain time and temperature. The environment temperature was different from the actual curing reaction temperature. The reaction temperature has time delay compared with the environment temperature. Moreover, the greater the heating rate, the greater the thermal effect of curing reaction in the unit time, the greater the temperature difference, the delay effect is more obvious. So the peak temperature is also higher.
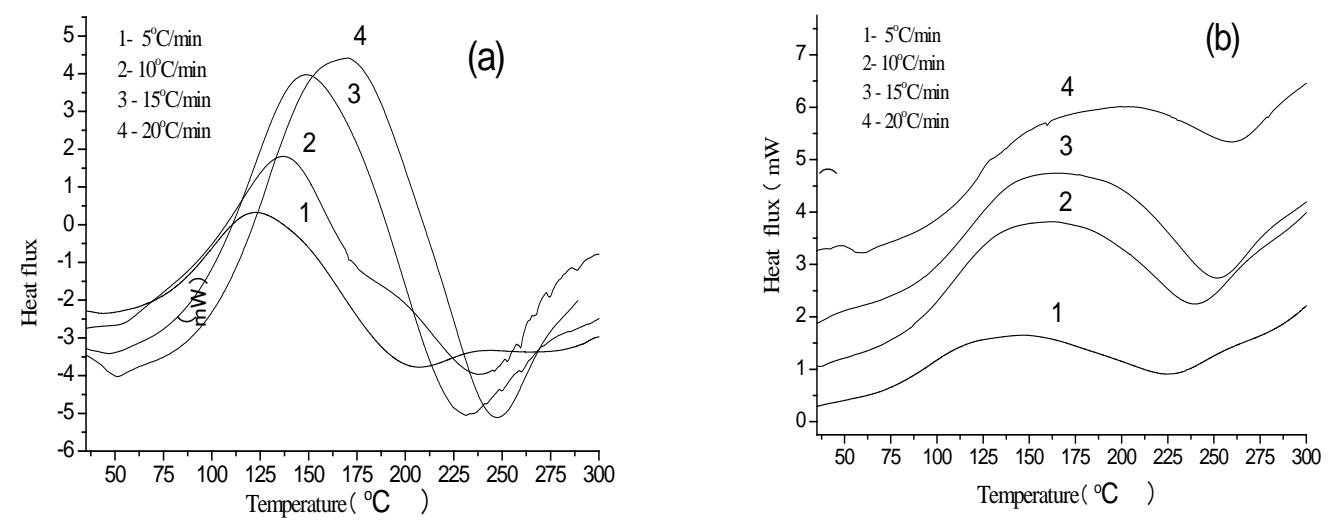

Figure 1. DSC curves of different heating rate (a) epoxy resin, (b) epoxy asphalt

The exothermic peak of epoxy asphalt curing reaction is relatively flat compared with epoxy system. The reason is that the curing reaction is not a simple reaction and it's a comprehensive of multiple complex reactions [7]. Only the appearance showed a single exothermic peak, asphalt has a very complex composition as a kind of polymer compounds. The exist of carboxyl, phenolic hydroxyl groups in the asphalt will react with the epoxy groups [8-9], all of these make curing reaction of epoxy asphalt more complicated. Therefore, it shows that the exothermic peak is 
relatively flat. While the composition of epoxy resin and curing agent are relatively single, the reaction is faster, so it shows that the exothermic curve is sharper.

\subsection{The Relationship between Curing Degree and Temperature at Different Heating Rates}

The curing degree is defined as an epoxy curing reaction heat divided by the total heat at a certain time. The relationship between the curing degree and temperature of epoxy resin and epoxy asphalt was shown in Fig. 2 at different heating rates. It could be seen from the figures the two relationships exhibit very similar changes with the typical characteristics of self-catalytic reaction. At the beginning of curing, the curing degree increases slowly, which increase with the basic linear in the medium term, the speed is relatively fast, which increase slowly in the last stage. The reason is that in the initial stage of reaction epoxy asphalt curing system was in the transition phase from the liquid to the gel, the curing reaction is slower. In the curing medium resin began to form a cross-linked network structure, from the gel to the glass state, the crosslinking density increased rapidly, and the curing reaction was relatively fast. The structure system of the crosslinking network was basically formed in the late stage of curing, and the viscosity of the curing system was relatively large. At the time, it became very difficult to "impact" of the macromolecules, the curing reaction was controlled by chemical control into diffusion, and the curing rate decreased obviously.

In addition to, with the heating rate from $5{ }^{\circ} \mathrm{C} / \mathrm{min}$ to $20{ }^{\circ} \mathrm{C} / \mathrm{min}$, the temperature wherever the epoxy and epoxy asphalt system to reach the same curing degree is gradually increased. The difference is in the same curing degrees, the temperature of epoxy asphalt system is higher than epoxy system. The reason is that with the raise of heating rate, the increase of reactants crosslinking density make reactant monomer not be diffused into the gel structure in time. Resulting in later curing reaction temperature is increased, and the effect of diffusion on the epoxy asphalt is more obvious during the curing process.
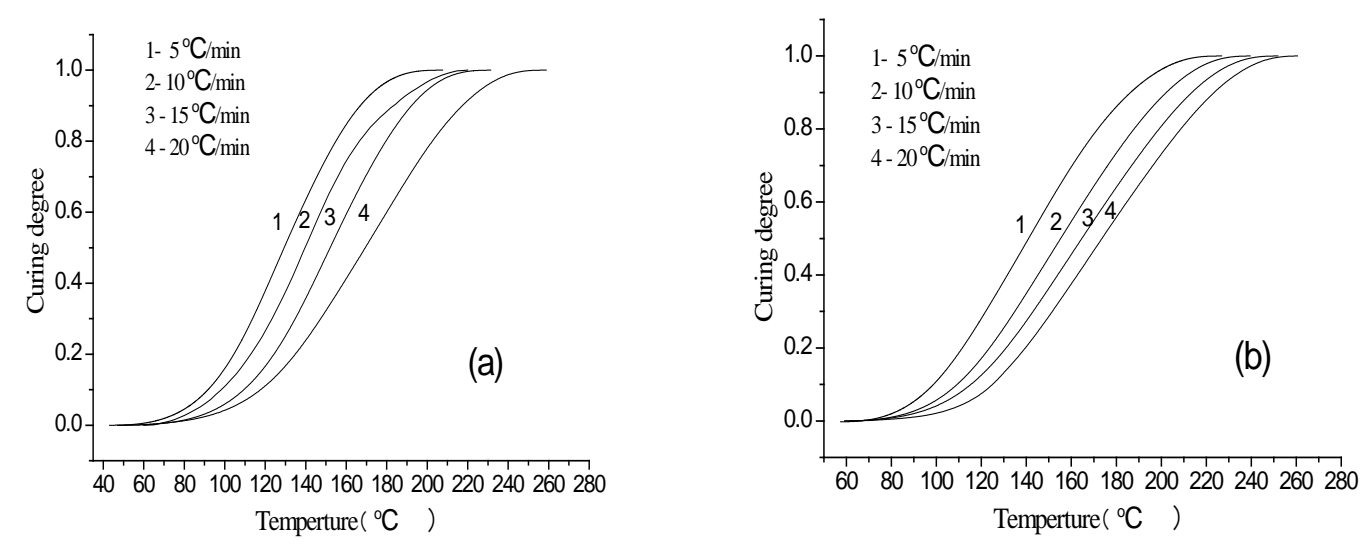

Figure 2. Curves of curing degree vs temperature of different heating rate (a) epoxy resin, (b) epoxy asphalt

\subsection{Calculation of Activation Energy}

According to Kissinger equation [10], during the curing of epoxy resin and epoxy asphalt, the relationship between peak temperature and heating rate is as follows:

$$
\mathrm{d}\left[\ln \left(\beta / T_{\mathrm{p}}^{2}\right)\right] / \mathrm{d}\left(1 / T_{\mathrm{p}}\right)=-E_{\mathrm{a}} / R
$$

In the Equation (1), $\beta$ is heating rate, $T_{\mathrm{p}}$ is a non-isothermal DSC curve peak temperature, $E_{\mathrm{a}}$ is the activation energy of reaction, $R$ is the ideal gas constant.

The DSC curves peak temperatures of the epoxy and the epoxy asphalt system at different heating rates were converted into the Kissinger equation, $\operatorname{Ln}\left(\beta / T_{\mathrm{p}}{ }^{2}\right)$ to $\left(1 / T_{\mathrm{p}}\right)$ mapping, as shown in Fig. 3. The obtained results were linear regression, the apparent activation energy is obtained by the slope of straight line, and the results were shown in Table 1. From those we can conclude that the activation energy of the reaction was increased after adding asphalt and the curing rate decreased. The reason is that the existence of the asphalt molecules, which makes the reactant molecules dispersed, and the probability of contact with each other is reduced. With the increase of 
the viscosity of curing system, the curing reaction is gradually from chemical control transformed into diffusion control. In the crosslinked network structure, block effect of asphalt is dominant.

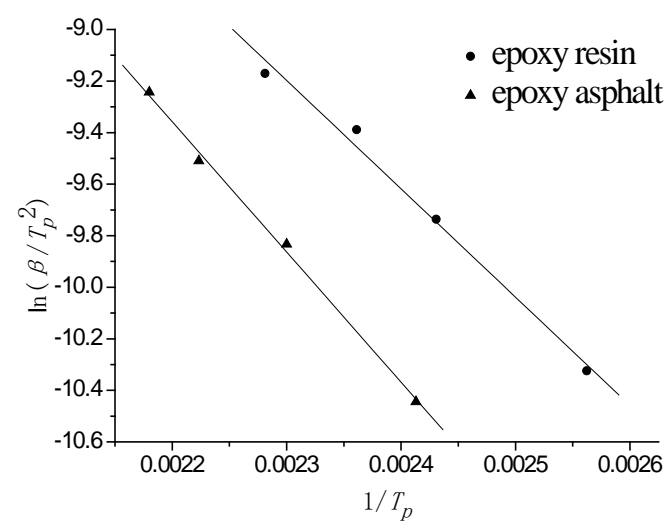

Figure 3. Activation energy of reaction by Kissinger method Table 1. Activation energy of two kinds of curing system

\begin{tabular}{|l|l|l|l|}
\hline system & linear equation & $E_{a} /\left(\mathrm{kJ} \cdot \mathrm{mol}^{-1}\right)$ & $R^{2}$ \\
\hline epoxy resin & $Y=0.47-4203.44 x$ & 34.95 & 0.9958 \\
\hline epoxy asphalt & $Y=1.76-5053.87 x$ & 42.02 & 0.9985 \\
\hline
\end{tabular}

\subsection{Self-Catalytic Reaction Model}

The phenomenological model was used to study most of reaction of the resin curing kinetics. Because this model avoided the types and details of chemical reaction during curing and its form is relatively simple. Through the model fitting curing process, this is in accordance with a kinetic model, and then obtain the kinetic parameters [11]. The study object of the self-catalytic reaction model is a macroscopic whole curing reaction. Most of the reaction mechanism of amine-epoxy system could be described by the model, which could be expressed by Kamal equation [12].

$$
d \alpha / d t=\left(k_{1}+k_{2} \alpha^{m}\right)(1-\alpha)^{n}
$$

In the Equation (2), $m$ and $n$ are the reaction order; $k_{1}$ and $k_{2}$ are non-catalytic reaction rate constant and self-catalytic reaction rate constant respectively. The relationship between curing degree and time of different heating rate was shown in Fig. 4.
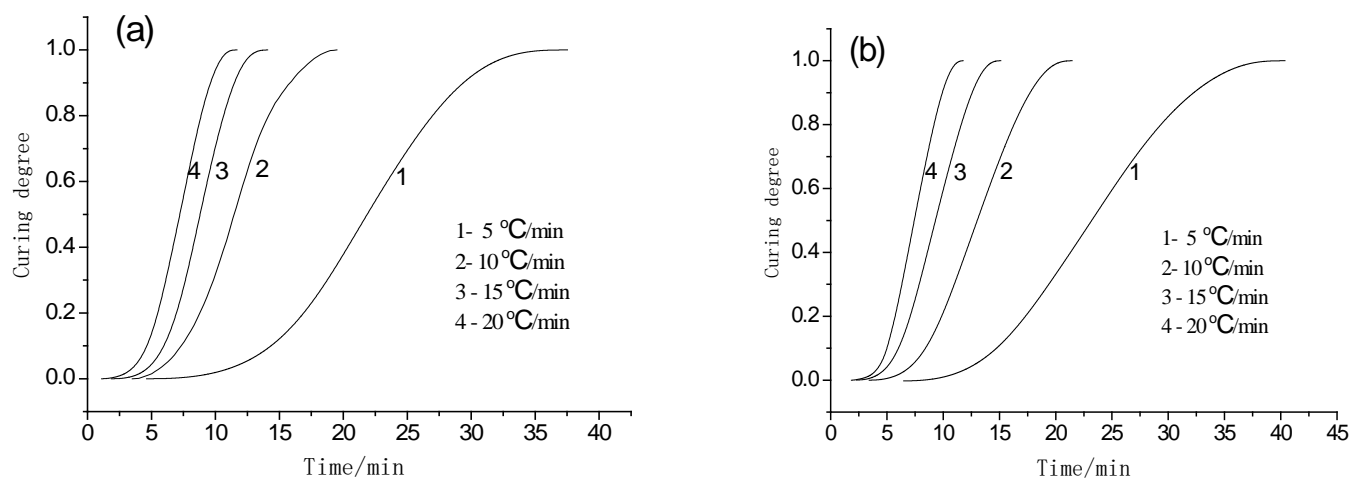

Figure 4. Curves of curing degree and time for different heating rate (a) epoxy resin, (b) epoxy asphalt

The kinetic parameters $\left(k_{1}, k_{2}, m, n\right)$ were fitted by the least square method, which were listed in Table 2. It could be seen that the non-catalytic reaction rate constant $k_{1}$ of the two curing systems were basically the same. But for the self-catalytic reaction rate constant $k_{2}$, at the same heating rate, the epoxy asphalt system is less than the epoxy system. The epoxy asphalt system reaction order of $m$ and $n$ were less than epoxy system. The above shown that the curing rate of epoxy asphalt system is less than that of the epoxy system, the concentration has little effect on epoxy asphalt system. Thus, it's further verified that the modified amine curing reaction is indeed a self-catalytic reaction. 
Table 2. Non isothermal curing kinetic parameters of Kamal model fitting

\begin{tabular}{|c|c|c|c|c|c|c|}
\hline $\begin{array}{c}\text { curing } \\
\text { system }\end{array}$ & $\begin{array}{c}\text { heating reat } \\
{ }^{\circ} \mathrm{C} / \mathrm{min}\end{array}$ & $\begin{array}{c}\text { rate constant } \\
k_{1} / \mathrm{min}^{-1}\end{array}$ & $\begin{array}{c}\text { rate constant } \\
k_{2} / \mathrm{min}^{-1}\end{array}$ & $\begin{array}{c}\text { reaction } \\
\text { order }(m)\end{array}$ & $\begin{array}{c}\text { reaction } \\
\text { order }(n)\end{array}$ & $\begin{array}{c}\text { reaction order } \\
(m+n)\end{array}$ \\
\hline \multirow{2}{*}{$\begin{array}{l}\text { epoxy } \\
\text { resin }\end{array}$} & 5 & 0.0010 & 0.1737 & 0.7294 & 0.6874 & 1.4168 \\
\cline { 2 - 7 } & 10 & 0.0012 & 0.4136 & 0.8876 & 0.8729 & 1.7605 \\
\cline { 2 - 7 } & 15 & 0.0010 & 0.4775 & 0.7528 & 0.6621 & 1.4149 \\
\cline { 2 - 7 } & 20 & 0.0010 & 0.4731 & 0.7026 & 0.5909 & 1.2935 \\
\hline \multirow{3}{*}{$\begin{array}{c}\text { epoxy } \\
\text { asphalt }\end{array}$} & 5 & 0.0012 & 0.1196 & 0.5668 & 0.5985 & 1.1653 \\
\cline { 2 - 7 } & 10 & 0.0010 & 0.2251 & 0.5958 & 0.5642 & 1.1600 \\
\cline { 2 - 7 } & 15 & 0.0010 & 0.3197 & 0.6109 & 0.5487 & 1.1596 \\
\cline { 2 - 7 } & 20 & 0.0010 & 0.4202 & 0.6007 & 0.5523 & 1.1530 \\
\hline
\end{tabular}

\section{Conclusion}

With the increase of heating rate, the exothermic peaks of DSC curves of epoxy and epoxy asphalt system are gradually moved to the high temperature region. And, the peak temperature of epoxy asphalt is higher than epoxy resin. At the same time the exothermic peak is relatively flat.

The curing reaction of epoxy resin and epoxy asphalt is a typical self-catalytic reaction. The change law of curing degree and temperature is very similar, which could be divided into three stages. At the late of curing reaction, the reaction type is changed from the chemical control stage to the diffusion control stage.

The apparent activation energy of epoxy system and epoxy asphalt system are $34.95 \mathrm{~kJ} / \mathrm{mol}$ and $42.02 \mathrm{~kJ} / \mathrm{mol}$ respectively. The introduced asphalt into epoxy curing system decreased the curing reaction rate. At the late of curing reaction, it was dominated by diffusion control.

The self-catalytic reaction rate constant $k_{2}$ and the reaction order $m$ and $n$ of the epoxy asphalt system are all slightly less than the epoxy system, however, the non-catalytic reaction rate constant $k_{1}$ is almost the same. That has mentioned above shows that the reaction mechanism of the two is similar. It is further verified that the curing reaction rate of epoxy asphalt system is lower than epoxy system, and they are both self-catalytic reaction.

\section{Acknowledgement}

The research was sponsored by the Special Fund for Basic Scientific Research of Central College, Chang'an University (Project No 310821162009).

\section{References}

[1] Yildirim Y. "Polymer modified asphalt binders”, Construction and Building Materials, 2007, 21, pp.66-72.

[2] Zhou Wei, Zhao Hui, Wen Jun, et al. "Study on effects of flexible curing agent on the structure and performance of epoxy asphalt”, Journal of Wu han University of Technology, 2011, vol33, 7, pp. 28-31.

[3] Xie Hongfeng, Dai Jie, Liu Chengguo, et al. "Thermal analysis of epoxy asphalts”, Polymer Materials Science and Engineering, 2009, vol25, 11, pp. 115-117.

[4] Qian Yuchun, Chen Shuanfa, Cong Peiliang. "Curing characteristics of epoxy asphalt studied by differential scanning calorimetry”, Journal of Wu han University of Technology, 2011, vol33, 11, pp. 24-27.

[5] Cao Xuejuan, Tang Boming. "Study on cure reaction condition of epoxy asphalt concrete by thermal analysis kinetics”, Journal of Highway and Transportation Research and Development, 2008, vol25, 7, pp. 17-20. 
[6] Nie Hao, Zou Xiaoling, Xie Zhen. "HDP epoxy asphalt mixture curing reaction kinetics analysis”, Highway Engineering, 2014, vol39, 3, pp. 294-297.

[7] Kong Donglei. "Study on the curing kinetics and the acoustic-speed characteristics of epoxy asphalt mixture”, Nan jing, Southeast University, 2012.

[8] J.L.Goodrich, L . Calif, R,J.Statz, et al, "Polymer and asphalt reaction process and Polymer-linked-asphalt product “, USP, 5306750, 1994.

[9] Statz R J, Griffin E R. "Epoxy functionalized ethylene copolymer asphalt reaction products”, USP, 0087997, 2003.

[10] Lee JaeYoung, Shim MiJa, Kim SangWook. "Effect of modified rubber compound on the cure kineties of DGEB/MDA system by Kissinger and iseonversional methods”, Thermochmica Acta, 200l, 371, pp. 45-51.

[11] Zhang Jing, Huang Pei. "Research Advances in Epoxy Resin Curing Kinetics”, Materials Review, 2009, vol23, 7, pp. 58-61.

[12] Sourour S, KamalM R. "Differential scanning calorimetry of epoxy cure:isothermal cure kinetics”, Thermochimica Acta, 1976, 14, pp. 41-59. 